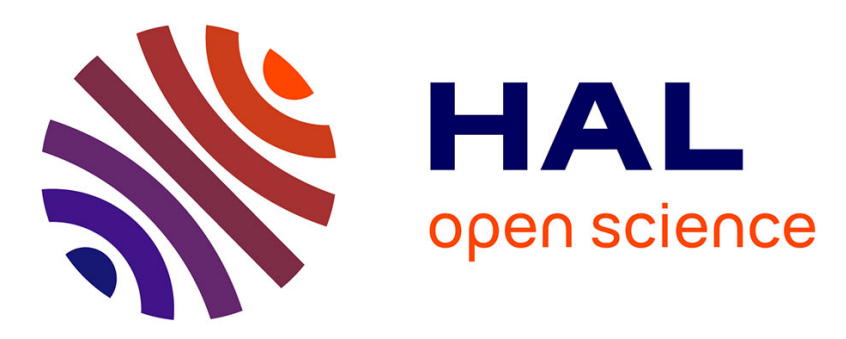

\title{
De la variation linguistique dans le prêche populaire mauritanien \\ Catherine Taine-Cheikh
}

\section{To cite this version:}

Catherine Taine-Cheikh. De la variation linguistique dans le prêche populaire mauritanien. Aleya Rouchdy. Language Contact and Language Conflict in Arabic. Variations on a Sociolinguistic Theme, Routledge Curzon, pp.177-203, 2002. halshs-00459704

\section{HAL Id: halshs-00459704 https://shs.hal.science/halshs-00459704}

Submitted on 24 Feb 2010

HAL is a multi-disciplinary open access archive for the deposit and dissemination of scientific research documents, whether they are published or not. The documents may come from teaching and research institutions in France or abroad, or from public or private research centers.
L'archive ouverte pluridisciplinaire HAL, est destinée au dépôt et à la diffusion de documents scientifiques de niveau recherche, publiés ou non, émanant des établissements d'enseignement et de recherche français ou étrangers, des laboratoires publics ou privés. 


\title{
De la Variation Linguistique dans le Prêche Populaire Mauritanien
}

\author{
Catherine Taine-Cheikh \\ CELLMA (CNRS - France)
}

Depuis l'étude pionnière de $\mathrm{H}$. Blanc (1960), un certain nombre d'auteurs se sont intéressés aux phénomènes de variation interne à l'arabe (du type arabe/ arabe). Elles ont été menées le plus souvent dans les pays du Moyen-Orient, car au Maghreb l'attention semble avoir été plus attirée par les cas d'alternance entre l'arabe et les langues européennes (français, anglais, etc.).

En Mauritanie, contrairement à un pays maghrébin comme l'Algérie et bien que le français $y$ fut longtemps langue officielle, le code-switching arabe/français est très peu développé jusqu'à présent. Les prêches que nous proposons d'étudier ici présentent une variation tout à fait étonnante à l'échelle de la société mais celle-ci se fait encore entièrement à l'intérieur de la langue arabe. Il est rare qu'un même locuteur ait recours aussi systématiquement à différentes variétés linguistiques et de ce point de vue on ne peut pas dire que O. Sidi Yahya soit représentatif des locuteurs mauritaniens. Ses discours religieux nous ont semblé cependant intéressants à étudier, car ils jouissent d'un grand succès public et sont typiques d'un contexte social relativement nouveau.

\section{LES PRECHES DE MUHAMMAD O. SIDI YAHYYA ET LEUR CONTEXTE}

Le personnage de O. Sidi Yahya, auquel Z. O. Ahmed Salem a consacré une partie de sa thèse (1996), occupe une place à part sur la scène mauritanienne contemporaine. Il est nécessaire, pour en comprendre le rôle, de préciser l'environnement sociologique et culturel dans lequel il intervient.

\section{Aperçu sur la société mauritanienne}

Pour Mokhtar O. Daddah, premier président de la Mauritanie (1960-78), ce pays situé au sud du Maroc et au nord du Sénégal, avait vocation à être un trait d'union entre l'Afrique noire et l'Afrique blanche. Même si les faits ne sont pas à 
la hauteur des espérances, cette représentation de la Mauritanie a l'avantage de souligner d'emblée le caractère multi-ethnique et pluri-culturel de la société. Arabophone, la population l'est dans sa majorité, mais il faut noter qu'environ 25 à $30 \%$ des Mauritaniens ne sont pas de langue maternelle arabe. Descendant des anciennes populations sédentaires du sud et du sud-est, ces populations négro-africaines appartiennent aux mêmes communautés que leurs voisins sénégalais ou maliens et parlent des langues du groupe ouest-atlantique, pulaar et soninké principalement, mais aussi wolof (notamment vers Rosso, dans l'extrême sud-ouest). Quant à la majorité arabophone composée de Maures (euxmêmes s'appellent al-biđ̛̣aan - d'un terme qui appartient à la même racine que l'adjectif abyađa "blanc"1 —), elle se caractérise d'abord par rapport au dialecte arabe hassaaniyya et à la culture orale qu'elle véhicule, même si l'un et l'autre sont inséparables d'un mode de vie traditionnel en voie de disparition. Encore largement nomades jusqu'au début des années 1970, les Maures se sont sédentarisés en masse dans les villes et villages, en particulier à Nouakchott et dans ses environs ${ }^{2}$. Les bouleversements socio-économiques ont influé sur l'organisation sociale ancienne mais sans mettre fin à l'existence des tribus et à la hiérarchie sociale qui caractérisaient la société bédouine maure. L'aspect le plus visible - et le plus choquant - réside dans la place qu'occupent respectivement les Maures "blancs" et les Maures "noirs" (hraațiin). Les premiers, au statut traditionnel de guerrier ou de religieux plus ou moins prestigieux, se sont globalement assez bien reconvertis dans la société moderne, car, en tant que groupes, ils gouvernent politiquement et économiquement le pays. Quant aux seconds, qui constituent peut-être la moitié de la population maure, ils sont la partie la plus défavorisée du pays et continuent, comme par le passé, à effectuer les tâches les plus dures physiquement, les moins prestigieuses et par là même les moins rémunératrices. L'iniquité de leur sort ne relève pas cependant de la couleur de leur peau, mais du statut social dont ils sont prisonniers, dans une société encore largement dominée par les valeurs traditionnelles.

\section{L'Islam et ses pratiques}

A l'Indépendance, le territoire anciennement colonisé par la France prit le nom de République Islamique de Mauritanie, la référence à l'Islam mettant en évidence l'un des points communs à l'ensemble de la population.

L'islamisation de l'Afrique de l'ouest a commencé très tôt (peut-être avec les Soninkés à l'époque du royaume de Ghana), mais on en retient surtout l'épisode almoravide, qui associe les Berbères de cette région à une page glorieuse de l'Afrique du Nord. Après le XIè siècle, l'islamisation a dû se poursuivre, tandis que le mouvement d'arabisation dialectale ne commençait lui qu'au XIVè siècle, après l'arrivée des Arabes $\mathrm{Ma}^{\mathrm{q}} \mathrm{qil}$ au Sahara occidental. Ce que les traditions ont vulgarisé, en particulier dans le sud-ouest du pays (théâtre, au XVIIè siècle, d'un

${ }^{1}$ Cet ethnoterme a deux extensions différentes selon qu'il englobe ou non les anciens esclaves ou Maures "noirs", cf. C. Taine-Cheikh (désormais C. T.-C.), 1989.

${ }^{2}$ Fondée en 1957 pour devenir la capitale du futur pays, Nouakchott a attiré en quarante ans entre le tiers et la moitié de la population globale (au moins 800000 habitants sur un total d'environ deux millions et demi de personnes). 
affrontement entre deux coalitions tribales, l'une à dominance arabe, l'autre à dominance berbère), c'est l'opposition entre les tribus guerrières considérées comme d'origine arabe, et les tribus maraboutiques, qui seraient principalement d'origine berbère.

Nous en retiendrons que, dans la société maure traditionnelle, la religion islamique est à la fois l'affaire de tous et celle d'un groupe en particulier, celui des marabouts (tolba) ${ }^{3}$. Dans les écoles supérieures traditionnelles (mahađ̛̣ra-s), que les tribus maraboutiques administraient et étaient seules à fréquenter, l'apprentissage du Coran se prolongeait par une étude approfondie de toutes les sciences islamiques, parmi lesquelles celle de la jurisprudence tenait une place très importante. Pour être un bon musulman, il fallait en effet respecter l'ensemble des obligations cultuelles, mais un soin tout particulier était accordé aux prières et à la connaissance précise des rituels à observer. Initié aux fondements du rite malékite dans l'Abrégé (muxtașar) d'al-Akhdariy, le jeune lettré passait ensuite à l'apprentissage par cæur de l'Abrégé (muxtașar) de Khalil, du XIVè siècle, ou de la Lettre (risaala) d'Ibn Abi Zayd - l'un des plus anciens résumés du rite - avant de compléter éventuellement sa formation par l'étude du Livre de la Purification (kitaab al-tahaara) d'Ibn 'Aašir où sont détaillées avec une extrême minutie tous les comportements à suivre pour éviter qu'une mauvaise gestion des humeurs corporelles n'entâche les prières de nullité ${ }^{4}$.

Les non-lettrés étaient donc généralement considérés comme de piètres musulmans, en dehors de l'élite des guerriers ${ }^{5}$. Ceci est particulièrement vrai des hraațiin qui manquaient autant d'instruction que de temps pour avoir une bonne pratique religieuse.

La prière du Vendredi fut l'objet de discussions juridiques, car la vie bédouine n'était pas compatible avec l'obligation de se réunir à la mosquée. Même dans les villes anciennes où la condition du quorum minimum pouvait être remplie (cf. A. J. Wensinck, article "Khuṭba" dans l'E.I.), la réalité du prêche ne semble pas s'être imposée, faute peut-être d'un pouvoir temporel reconnu, au nom duquel celui-ci aurait pu être prononcé. Le prêche n'a donc pris de l'importance en Mauritanie que depuis l'Indépendance, avec l'instauration d'un pouvoir d'Etat islamique et avec la sédentarisation de la population.

\section{Les prêcheurs de Nouakchott et leurs publics}

Actuellement, dans la capitale et ses environs, on compte un grand nombre de mosquées où les hommes adultes se rendent en masse, notamment pour la prière du Vendredi. La grande mosquée officielle, dont les prêches sont retransmis à la radio, est la mosquée dite "saoudienne" (en raison de l'origine de son financement), érigée dans le centre de Nouakchott au début des années 1980.

${ }^{3}$ Le pouvoir qu'ils exerçaient dans la société reposant en grande partie sur leur connaissance des choses de l'au-delà et sur leur lien privilégié avec le surnaturel (sur "l'administration de l'invisible", cf. A. W. O. Cheikh, 1985: 933 et sq.).

${ }^{44}$ De ce point de vue il faut sans doute noter l'influence ancienne de l'islam confrérique sur les pratiques religieuses des Mauritaniens, mais il n'y a pas lieu de préciser les différences liées aux trois grandes confréries représentées dans le pays (la qaaḍiriyya, la šaađ̣iliyya et la tižaaniyya).

${ }^{5}$ Le maǵvri est, dit-on, un homme d'honneur et de religion, le hassaani, un homme d'honneur sans religion et le hscysiini, un guerrier sans honneur ni religion. 
Seuls les prêcheurs officiels ont droit à une petite subvention annuelle, mais leurs orientations semblent relativement variées. Plusieurs mosquées subventionnées sont animées par des islamistes, bien que le parti islamiste de la ${ }^{2}$ umma n'ait pas été reconnu par le gouvernement. Elles ne sont donc pas en marge de l'islam officiel comme celle de Teyaaret, construite sur un terrain squatté par O. Bunyamiin (dont l'Etat a fini par lui donner propriété) ${ }^{6}$ ou celle de RyaaD, considérée comme rebelle bien qu'elle se dise apolitique ${ }^{7}$.

Depuis trois ans, l'un des prêcheurs les plus recherchés est celui de Tevragh Zeyna, qui traite des problèmes de société (luxe, prostitution, viol, ...) dont l'importance nouvelle frappe les esprits. Malgré cette ferveur récente pour un nouveau venu, O. Sidi Yahya (désormais O. S. Y.), qui introduisit la mode du prêche populaire en Mauritanie, continue à occuper une place unique en son genre.

Prêcheur auto-proclamé qui ne bénéficie pas de l'attribution officielle d'une mosquée, O. S. Y. a commencé à se faire connaître dans les années 1985 ou 86. Son public de prédilection, celui qui assiste à ses prêches réguliers du lundi ou va écouter ses interventions lorsqu'il se déplace à travers tout le pays, est un auditoire populaire, composé de petites gens d'extraction modeste (les hraațiin y sont majoritaires), peu instruits et récemment urbanisés. Cependant, si sa notoriété est importante, c'est parce que ses discours, enregistrés sur cassettes, sont diffusés à longueur de journée dans les marchés et les lieux publics. Ses prêches ont pu ainsi susciter des discussions spontanées à une époque où la "démocratisation" de la vie politique n'avait pas encore facilité la tenue de débats publics.

Pour Z. O. Ahmed Salem, la libéralisation de la presse et l'ouverture au multipartisme ont contribué, à partir de 1992, à affaiblir la portée contestataire de ses discours et l'ont poussé dans la voie d'une radicalisation politique qui lui a fait perdre une partie de sa base (il est le président du hizb, le parti non reconnu de la ${ }^{2} u m m a$ ). On trouvera dans son travail (1996: 207 et sq.) une analyse du parcours idéologique de $O$. S. Y. et de ses relations avec le mouvement néo-fondamentaliste, malgré une certaine ressemblance avec les "nouveaux intellectuels islamistes" qui, comme lui, font figure de prêcheurs libres, en marge des milieux islamiques officiels.

Notre propos, complémentaire de celui de O. Ahmed Salem, est d'étudier concrètement la langue utilisée dans les prêches afin de savoir si les choix langagiers sont explicables et s'ils jouent un rôle dans le succès de l'orateur.

${ }^{6}$ O. Bunyamiin apparaît comme un traditionaliste d'une tendance très spéciale. D'une part il refuse toute aide de l'Etat - façon chez lui de marquer sa non-reconnaissance du pouvoir officiel - . D'autre part il interdit l'entrée de sa mosquée aux fumeurs, aux prédicateurs $\left(d u^{\complement} a a t\right)$ et aux anciens esclaves (sauf à prouver que leurs deux parents ont été officiellement libérés par leurs maîtres), ne craignant pas, là encore, de placer son interprétation de la loi islamique au-dessus des lois officielles !

${ }^{7}$ La mosquée "de l'appel et de la transmission", žamaa ${ }^{\varsigma} a t$ al-da $w a$ wa-l-tabliig, fait du prosélytisme en faveur d'un mouvement d'origine pakistanaise proche de l'islamisme. 


\section{LES DIFFERENTES VARIETES LINGUISTIQUES EN PRESENCE}

Au cours de nos travaux de recherche sur la Mauritanie, nous avons rencontré trois variétés d'arabe et il nous semble possible de les définir avec plus ou moins de précision, tant linguistiquement que sociolinguistiquement. On peut donc parler de triglossie, en reprenant le terme utilisé par A. Youssi pour décrire une situation relativement proche (1983).

\section{L'arabe dialectal}

Le seul dialecte arabe parlé en Mauritanie est le hassaaniyya. Son extension dépasse même largement les frontières, en particulier vers le nord où la région de Goulimine, dans le sud marocain, reste encore la zone de transition décrite par V. Monteil (1948) - quelques milliers de hassanophones y sont en contact avec des populations essentiellement berbérophones - Un élément nouveau apparaît cependant pour les hassanophones marocains, celui de la pression qu'ils subissent de la part de la dariža (ou "arabe marocain") et qui tend à s'imposer à tous, notamment par la radio, pour peu que les liens avec la communauté d'origine se distendent (cf. C. T.-C., 1997).

La comparaison avec le Maroc est intéressante, car elle fait mieux ressortir la spécificité de la Mauritanie. Dans le royaume chérifien qui se caractérisait auparavant par un grand nombre de variétés dialectales ${ }^{8}$, l'évolution récente ne modifie pas seulement les rapports entre l'arabe et le berbère, elle agit aussi fortement sur les parlers arabes en nivelant les différences et en favorisant l'émergence de formes dominantes (celles-ci pouvant être aussi bien des formes neutres que des formes marquées, telles l'usage du fém. de 2è pers. comme forme commune de l'inaccompli au masc. et au fém. sing.).

En Mauritanie, curieusement, nous n'avons aucune trace de véritable différenciation dialectale. Cela ne signifie pas que les hassanophones ne détectent pas entre eux l'origine géographique de leurs interlocuteurs mais, s'ils le font, c'est en fonction de facteurs marginaux qui n'affectent en rien le système linguistique du hassaaniyya. En effet, en dehors de ce qu'on appelle "l'accent", les différences portent sur le lexique et notamment sur les emprunts".

Les deux macro-discriminants de la dialectologie arabe que sont pour nous les interdentales et le qâf (cf. C. T.-C., article à paraître dans $M A S \mathrm{n}^{\circ}$ 9) n'ont pas d'effet à l'échelle de la Mauritanie: les interdentales sont partout conservées et le qâf se réalise toujours comme une sourde [g]. La seule différence notable qui oppose l'est et le nord-est (pays "de montagne") au reste du pays maure (pays "de sable", dont le cœur est situé dans la Gebla, au sud-ouest) concerne la

${ }^{8}$ Depuis G. S. Colin (cf. notamment l'article "al-Maghrib" dans l'E. I.), on classe les parlers arabes marocains en citadins, montagnards et bédouins, sans oublier la distinction entre parlers juifs et parlers musulmans - manière d'essayer de comprendre comment l'histoire a pu façonner une aussi grande diversité - . Sur ce thème, voir les actes du colloque Peuplement et arabisation au Maghreb occidental. Dialectologie et histoire, édités par J. Aguadé, P. Cressier et A. Vicente, en 1998.

${ }^{9}$ Dans les villes anciennes de l'est, on trouvera plus d'emprunts au soninké, voire au bambara; dans le sud-ouest domineront les emprunts au berbère zénaga ; dans la Sagya al-Hamra et le Rio de Oro (anciennement colonisées par l'Espagne), les emprunts au français sont remplacés par des emprunts à l'espagnol. Sur le lexique du ḥassaaniyya, cf. C. T.-C., 1988-98. 
réalisation du gayn ${ }^{10}$; il est toujours réalisé comme une occlusive uvulaire chez les premiers alors que les seconds réalisent normalement une fricative $[\dot{g}]$ sauf s'il s'agit d'une géminée ([qq] pour $/ \dot{\mathrm{g} g} /$ ). On peut peut-être, dans ce ġayn fricatif, voir une influence du berbère zénaga qui survécut au sud-ouest beaucoup mieux qu'ailleurs ${ }^{11}$. Dans ce cas, la réalisation occlusive du gayn (qu'on retrouve d'ailleurs dans un certain nombre de dialectes bédouins, au sud de l'Algérie et au nord de la péninsule arabique notamment) pourrait être un trait très ancien et pas seulement le produit d'une évolution interne, certes logique mais propre aux tribus de l'est.

Si l'unité du dialecte hassaaniyya est étonnante et, nous semble-t-il, exceptionnelle dans le monde arabe (compte tenu, non pas du nombre de locuteurs - probablement pas plus de 2 millions au total - , mais de la surface concernée en $\mathrm{km}^{2}$ ), il faut noter que nous n'avons aucune preuve de l'existence de parlers dialectaux arabes avant l'arrivée des $\mathrm{Ma}^{\mathrm{S}}$ qil alors que le nom même de hassaaniyya, qui fait référence à l'ancêtre de la fraction des Bani-Ḥassaan, nous pousserait plutôt à en rejeter l'hypothèse ${ }^{12}$. A l'image de cette paternité "unique", on ajoutera celle d'une population nomade, donc en constants déplacements, ce qui peut se comprendre comme "en état d'échanges constants" - la sédentarisation ayant souvent correspondu a contrario dans le passé à une tendance marquée à la différenciation - .

Enfin il faut dire que la correspondance observée en général entre population de nomades et dialecte de type bédouin se vérifie parfaitement. On a affaire à un parler qui, tout en offrant les caractéristiques communes aux parlers maghrébins (1ère pers. sing. de l'inaccompli en $n$ - et chute des voyelles brèves en syllabe ouverte, notamment), se caractérise clairement comme un dialecte conservateur $^{13}$. Souvent à mi-chemin entre les parlers bédouins orientaux et les parlers de sédentaires maghrébins pour la simplification des oppositions morphologiques (ainsi celle du masc./ fém. dans les systèmes verbal et nominal), il va clairement de pair avec les premiers quand il s'agit de la syntaxe et du maintien des constructions anciennes (celle de l'état d'annexion est la plus visible, mais elle est loin d'être la seule).

\section{L'arabe littéraire}

Dans la société maure traditionnelle, comme dans l'ensemble du monde arabe, la majorité des biđaaan n'avaient pratiquement pas de contact avec l'arabe classique, en dehors des rudiments d'arabe coranique nécessaires à la profession de foi et à la prière. Nous avons vu précédemment qu'il y avait cependant un

${ }^{10}$ A noter qu'une différence curieuse dans la nomination des points cardinaux (C. T.-C., 1991a) suit approximativement les mêmes règles de répartition.

11 Sur le système consonantique du zénaga, encore parlé par quelques milliers de locuteurs bilingues, cf. C. T.-C., "Le zénaga de Mauritanie à la lumière du berbère commun", 1999.

${ }^{12}$ Quand H. T. Norris (1986) laisse entendre, à partir du récit d'Ibn Baț̣uuțah, qu'on parlait arabe à Oualata au XIVè siècle, il ne souligne pas assez nettement que les commerçants berbères pouvaient se faire comprendre du voyageur arabe grâce à leur connaissance de l'arabe classique ou plus vraisemblablement du dialectal marocain, sans qu'on puisse pour autant parler d'arabisation.

13 Pour une description globale du dialecte, cf. D. Cohen, 1963 et C. T.-C., 1988-98, Introduction au vol. 1. Pour une caractérisation du parler comme dialecte de nomades maghrébins, cf. C. T.-C., 1991b. 
groupe important de la société chez qui les études tenaient une grande place ${ }^{14}$. De ce point de vue la société maure fait là encore figure d'exception dans le monde arabe car, dans l'opposition générale entre nomades et sédentaires, les bédouins font plutôt figure d'ignorants par rapport aux seconds.

Appliqué à ce contexte, le concept de diglossie semble prendre tout son sens. D'une part on a bien affaire, avec l'arabe littéraire et le hassaaniyya, à "deux états d'une même langue, assez différents pour que la connaissance de l'un n'implique pas l'autre, assez semblables pour que la connaissance de l'un facilite considérablement l'acquisition de l'autre" (W. Marçais, [1930] 1961: 88), d'où l'intérêt de la notion de diglossie à côté du terme général, non spécifique, de bilinguisme. D'autre part ces deux variétés ont chacune des fonctions qui leur sont propres. Pour W. Marçais, qui fut le premier à tenter une description concrète de la diglossie arabe, la situation oppose au Maghreb un arabe écrit faisant office de langue de civilisation pour l'islam — "stylisé, normalisé, par un énorme effort philologique de grammairiens, de lexicographes, de collecteurs de vieilles poésies" (idem: 83) — et un arabe parlé qui diverge d'une région à l'autre, mais qui partout et toujours constitue le seul arabe utilisé dans la conversation courante.

Concernant le passé, et notamment les lettrés maures, cette analyse nous semble assez juste car la polarisation oral/ écrit $\mathrm{y}$ était effectivement fondamentale. Il faut cependant préciser que le rapport à l'écrit y était largement médiatisé par la voix et passait moins par l'œil que par l'oreille, les textes en arabe littéraire étant moins lus que récités, d'où l'opposition que nous avons proposée récemment (C. T.-C., "Langues, savoirs et pouvoir en milieu maure", 1998) entre l'oralisation de la culture populaire en dialecte et "l'auralisation" de la culture savante en arabe littéraire. Est-ce à dire que les lettrés ne parlaient jamais en arabe littéraire ? En quelque sorte, puisque même dans les mahađ̛̣ra-s c'était le dialecte qui était principalement utilisé dans l'enseignement pour l'explication des mots et les commentaires ${ }^{15}$. D'un autre côté, il faut voir qu'ayant souvent mémorisé des centaines, voire des milliers de pages, versifiées ou non, ils pouvaient au sens propre "parler comme dans les livres". Etant capables de composer, avec plus ou moins de spontanéité, des poèmes en arabe classique, on ne peut pas exclure qu'ils "improvisent" en prose. Cependant, il s'agissait d'une énonciation éminemment contrôlée, sur des thèmes relevant de certains domaines privilégiés (essentiellement les sciences islamiques) et dans une forme linguistique qui se devait d'être parfaitement conforme aux règles énoncées dans les grammaires ${ }^{16}$.

Cet arabe écrit oralisé (ou, mieux encore, cet arabe littéraire "auralisé") avait les normes connues de l'arabe classique et, de ce point de vue, on ne peut vraiment pas dire qu'il était "mal défini". Pourtant, dans la controverse qui a opposé A. S. Kaye (1972 et 1994) à S. A. El-Hassan (1978), nous aurions tendance à donner raison au premier, bien que ce soit le second qui soutienne, à

${ }^{14} \mathrm{Si}$ les femmes des tribus maraboutiques n'allaient guère, en général, au-delà de l'apprentissage du Coran, les hommes étudiaient souvent jusqu'à 25 ans et parfois beaucoup plus (al-Shaykh Sidiyya al-Kabiir s'instruisit loin de chez lui jusqu'à près de 50 ans avant de rentrer ouvrir sa mahađ̛̣̆ra, cf. A.W. O. Cheikh, 1991: 205).

${ }^{15}$ N'oublions pas que, chez les berbérophones, c'est le berbère zénaga qui était utilisé dans ce contexte, il y a peu de temps encore.

${ }^{16}$ La notion de faute linguistique était ancrée dans les esprits et entraînait, chez celui qui la commettait, une disqualification touchant à son honneur même. 
l'inverse d'A. Kaye, que l'arabe "mal défini" est peut-être le dialectal, mais certainement pas l'arabe littéraire. S'agissant en effet de la période contemporaine, nous n'avons plus affaire seulement à l'arabe littéraire classique, mais aussi à un autre arabe littéraire, plus détaché du modèle coranique et souvent appelé l'arabe moderne standard (AMS), sans qu'on sache très bien les différences acceptables de réalisation dues au passage de l'écrit à l'oral ${ }^{17}$ et sans qu'on prenne en compte les variations internes à l'AMS (tant écrit qu'oral), dues à l'interférence avec le dialecte maternel.

L'arabe littéraire étant plus facile à définir négativement que positivement ("It is much easier for the linguist to say what MSA is NOT than what IT IS", A. Kaye, 1972: 33), nous cernerons mieux l'AMS quand nous aurons vu ce qu'est l'arabe médian.

\section{L'arabe médian}

Dans la société contemporaine, les relations entre le dialecte et l'AMS correspondent moins aux rapports écrit/ oral établis par W. Marçais qu'à la situation décrite par C. Ferguson (1959). Dans son article "Diglossie", l'auteur américain redéfinit en effet ce concept en mettant l'accent sur les oppositions formel/ informel et haut/ bas ${ }^{18}$.

Le couple formel/ informel, moins tendancieux nous semble-t-il que celui de haut/ bas ${ }^{19}$, peut être compris par rapport aux circonstances d'énonciation, les situations officielles (discours politique ou scientifique, prêche, actualités télévisées, etc.) partageant avec l'écrit la propriété de favoriser le recours à l'AMS, alors que les situations non officielles (relations familiales ou de voisinage, conversations spontanées entre amis ou collègues de travail, etc...) rendent l'emploi de l'AMS peu probable. Mais la notion de "formel" renvoie aussi au fait que l'AMS, n'ayant pas de locuteurs natifs, ("MSA is not the mother tongue of any Arab", A. Kaye, 1994: 51), est une langue artificielle et idéelle à laquelle on n'a recours que sporadiquement, pour des raisons particulières.

En Mauritanie, les changements économiques et sociaux ont introduit de nouveaux centres d'intérêt, notamment avec l'ouverture sur le monde extérieur qui devint plus nette à partir des années 1960. L'influence des médias arabes et le développement des contacts avec les élites des autres pays donnèrent naissance à des mouvements intellectuels qui ressentirent la nécessité - et la possibilité - d'exprimer leurs idées sociales et politiques dans leur langue et non plus, comme ce fut le cas dans les premières années de l'Indépendance, en français. Il se développa donc, dans les années 1968-75, un nouvel usage de

17 Ainsi devrait-on distinguer, selon le linguiste libanais Anis Furayhah, le "Cultivated Spoken Arabic" qui est "basically MSA without any of the inflectional endings" (d'après A. Kaye, 1994: 49) du "Spoken MSA" proprement dit, qui ne se confond pas lui-même avec le "MSA of religious sermons", quasi assimilable au classique.

18 Il en découlera, chez des linguistes comme J. A. Fishman, l'idée de la diglossie comme un bilinguisme inégalitaire où les deux variétés ne sont pas nécessairement apparentées, ce qui a contribué à brouiller un peu plus les contours de ce concept.

${ }^{19}$ La minoration du dialecte par rapport à la langue littéraire n'est pas une nécessité et surtout, comme l'a par exemple montré M. H. Ibrahim (1986), le statut de variété prestigieuse n'est pas réservé au seul AMS mais peut fort bien, selon le contexte social, être associé à une variété dialectale. 
l'arabe, dont l'apparition est très liée aux mouvements politiques d'opposition, anti-gouvernementaux mais surtout anti-impérialistes et nationalistes, et qui s'implanta à la fois dans la jeunesse scolarisée des villes, largement francisante, et dans certains milieux intellectuels issus de l'enseignement traditionnel, mais influencés par le pan-arabisme.

Nous avons qualifié cet arabe de "médian" parce que, traduisant le terme arabe de wusțaa déjà en usage, il rendait bien compte de ses caractéristiques principales, à savoir le fait d'être plus ou moins à mi-chemin entre le dialecte et l'AMS (C. T.-C., 1978). D'une certaine manière, il semble correspondre à ce que de nombreux auteurs anglophones appellent Educated Spoken Arabic (ESA), c'est-à-dire l'arabe des éduqués, mais en fait il ne lui correspond sans doute que très partiellement - et pas seulement parce que, comme T. F. Mitchell l'a bien montré dans son article "What is educated spoken Arabic ?" (1986), l'ESA tend à couvrir plusieurs réalités relativement divergentes - En effet l'une des fonctions principales de l'ESA consiste, comme c'est souvent le cas en situation de diglossie pour les variétés intermédiaires (cf. P. Hawkins, 1983: 16), à opérer une "purification" linguistique, c'est-à-dire en l'occurrence à éliminer de la variété la plus basse les traits stigmatisants (la réalisation affriquée de tel phonème, la variante discontinue de la négation, etc., qui sont associés au parler non prestigieux d'une communauté locale, d'origine souvent rurale ${ }^{20}$ ). Ainsi l'élimination des caractéristiques trop particulières apparaît comme un point commun à l'ensemble des variétés d'ESA, sans qu'il soit apparemment nécessaire de distinguer très rigoureusement les variétés locales et les variétés interrégionale ${ }^{21}$. Pourtant la notion de stigmatisation va de pair, non pas tant avec la classicisation proprement dite, qu'avec le mouvement de koinéisation, selon la distinction mise en avant par H. Palva (1982) - distinction qui permet de comprendre pourquoi certains traits progressent (telle la réalisation $\left.{ }^{[}{ }^{1}\right] \mathrm{du} q \hat{a} f$ s'imposant au détriment d'un [q] ressenti localement comme typiquement druze), alors qu'ils sont en opposition avec la norme classique.

Cet aspect est à souligner car, dans la société maure (tant qu'il s'agit du moins d'un échange entre hassanophones), le problème ne se pose pas de faire un tri parmi les traits dialectaux puisque, comme nous l'avons vu précédemment, le hassaaniyya équivaut déjà à une forme dialectale koinéisée. L'arabe médian des hassanophones peut donc apparaître comme une variété semi-littéraire, comparable par bien des côtés à la variante dialectale classicisée étudiée par H. Palva (1969), mais elle reste sans doute plus proche du pôle purement dialectal que beaucoup de productions considérées comme caractéristiques de l'ESA, car elle n'a subi aucune "épuration"22.

${ }^{20}$ La constitution des parlers urbains semble illustrer aussi ce schéma, mais elle s'en distingue par le fait que certains traits locaux, même stigmatisés, peuvent se maintenir quand ils remplissent une fonction identitaire (cf. l'exemple jordanien étudié par H. R. E. Abd-el-Jawad, 1986).

${ }^{21}$ La notion de stigmatisation est très présente dans les travaux de T. F. Mitchell (cf. 1980 et 1986), qui concernent essentiellement les relations entre l'Egypte et le Levant - comme beaucoup de travaux menés à l'Université de Leeds grâce au Social Science Research Council dans les années 1980 -.

${ }^{22}$ Pour H. Palva, il est clair que la situation de contact croissant entre les dialectes locaux et l'arabe classique donne lieu à deux mouvements complémentaires de sens différent ("The traditional dichotomy is growing less clear-cut; in addition to the vertical style spectrum there is a horizontal development towards koineization of the dialects", 1969: 3), ce qui justifie la 
Les modifications observées en médian sont liées à l'introduction de lexèmes, empruntés à l'AMS pour les besoins de la communication et non pas, sauf rares exceptions, à une volonté de relever le style du discours en l'émaillant d'expressions classicisantes. La continuité entre l'arabe médian et l'arabe littéraire n'est pas assurée, car les emprunts sont traités essentiellement selon les règles de la morphologie du hassaaniyya. Par contre, elle l'est entre l'arabe médian et le dialecte, même si l'intégration des emprunts laisse subsister un certain nombre de caractéristiques phonologiques propres à l'arabe littéraire (respect de la distinction daad / đạa ${ }^{2}$, réalisation sourde [q] du qâf, maintien régulier des voyelles brèves en syllabe ouverte et maintien partiel du hamza). Par ailleurs les formes dialectales pures sont fréquentes et appartiennent à l'ensemble des classes syntaxiques, alors que les formes littéraires pures, nettement plus rares, relèvent essentiellement des catégories particulières (adverbes, particules, conjonctions, ...) qui échappent aux variations morphologiques.

L'arabe médian est donc bien une forme mixte, mais il est plus précisément, en Mauritanie du moins, un "dialecte mixte" au sens de P. Trudgill (1986: 59). Par ailleurs, si l'on peut considérer qu'il constitue maintenant un troisième code à côté de l'arabe littéraire et du dialecte, on se gardera bien d'affirmer que les codes et leurs fonctions se distinguent de manière aussi claire et tranchée dans la triglossie actuelle que dans la diglossie passée. Nous allons d'ailleurs voir, avec les prêches de O. S. Y., que les trois glosses peuvent se rencontrer dans un même discours.

\section{DE L'ALTERNANCE CODIQUE AUX FORMES MIXTES}

Bien qu'on ait affaire à un seul locuteur, en situation de quasi monologue, et à un public aux caractéristiques à peu près constantes, les variations observables dans les cassettes enregistrées sont extrêmement marquées. Nous allons maintenant les analyser en fonction de critères qui ont été établis pour les relations de langue à langue, mais qui s'appliquent aussi aux relations de dialecte à variété standard. La première distinction concerne l'opposition entre code-switching et code-mixing, mais on accordera également un traitement séparé aux différentes formes d'alternance codique.

\section{L'alternance codique séquentielle}

Le code-switching peut être défini comme le fait d'abandonner brusquement un code au profit d'un autre à un moment précis de l'énonciation ${ }^{23}$ mais, du point de vue purement linguistique, le fait de changer de code entre deux propositions ne pose pratiquement aucun problème. L'intérêt d'une telle alternance relève donc plutôt du domaine pragmatique (cf. P. Auer, 1986) que nous aborderons

distinction entre "koineized colloquial" et "semi-literary" (ou "elevated colloquial") faite par H. Blanc (1960: 85).

23 "By "code-switching" is meant a pattern of textual production in which a speaker alternates between continuous utterance segments in one language $\mathrm{L}_{\mathrm{x}}$ and another language $\mathrm{L}_{\mathrm{y}}$ with abrupt and clear-cut switching points, often at phrasal or clausal boundaries." (J. Heath, 1989: 23). 
dans le chapitre suivant. Il s'agit présentement de donner une illustration concrète du passage d'un code à un autre.

Pour faciliter l'analyse des exemples, nous adoptons deux styles différents: les italiques pour le pôle littéraire (AMS ou classique) et les normaux pour le pôle opposé (dialecte pur ou arabe médian).

(1) a. tartiibu -l-muṣhaf

moebduu ${ }^{2} u n$

bi-l-faatiha

a. ordonnancement le Livre commencé(MS) avec la Fatiha

a. "L'ordonnancement du Livre commence par la Fatiha

b. wa ammaa nuzuulu -l-quriaan aw tanazzulu

b. et quant à Révélation le Coran ou Révélation

b. et quant à la Révélation du Coran...ou comment le Coran

c. l-qur ${ }^{2} a a n$ fa inna-hu mobduu?un bi suurati

c. le Coran alors que-3MS commencé(MS) avec sourate

c. "fut révélé, et bien il commence par la sourate de

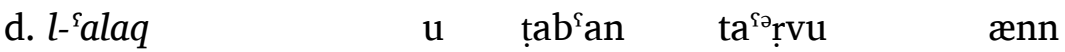

d. la goutte de sperme et bien sûr avez su(2P) que

d. "la goutte de sperme et bien sûr vous savez que

e. əl-quṛ aan maa-hu murattæb ḥasæb ən-nuzuul

e. le Coran nég.-3MS ordonné selon la Révélation

e. "le Coran ne suit pas l'ordre de la Révélation."

Dans ce premier cas nous avons clairement une période qui débute en AMS et s'achève en arabe médian. Du point de vue phonologique, l'opposition est peu marquée, car la fin de la phrase contient plusieurs formes empruntées à l'arabe littéraire: les substantifs nuzuul et tanazzulu "Révélation" (litt. "fait de descendre", noms d'action de 1ère et Vème formes) ainsi que qur?aan "Coran", l'adverbe tab $a{ }^{\S} a n$ "bien sûr", la préposition hascb "selon" et le verbe rattaba "ordonner". De toutes ces formes, seule la forme verbale est véritablement dialectalisée (en hassaaniyya, les participes passifs des formes dérivées sont en $m u$-, par opposition aux participes actifs qui sont en $m$-), car les autres emprunts sont intégrés pratiquement tels quels, notamment - dans le cas de hasceb et de nuzuul - en conservant leur voyelle brève en syllabe ouverte. Outre la forme participiale, les deux formes typiquement dialectales sont donc la négation nominale maa-hu et l'inaccompli $t a^{\text {S }} r v u^{24}$.

Quant au début de la phrase, son appartenance au pôle de l'AMS se manifeste:

- par l'ensemble du vocabulaire (et tout particulièrement l'emploi de ammaa ... fa inna-hu qui est typique de l'AMS),

- par la conservation des voyelles en syllabe ouverte, en particulier dans les noms,

- par une réalisation moins centrale des voyelles (préposition bi et non ba, article défini $a l$ et non al, conjonction $a w$ au lieu de $a w$ ), une conservation des voyelles longues finales (cf. ærmmaa) et des hamza,

${ }^{24}$ Sur ces différentes caractéristiques du dialecte mauritanien - passif en $u$-, négation du prédicat nominal et chute des voyelles brèves en syllabe ouverte, cf. C. T.-C., respectivement 1983, 1995-6 et 1988. 
- et surtout par la présence des finales désinentielles (marques casuelles en - $u$ du nominatif défini pour tartiibu, nuzuulu et tanazzulu, en -un du nominatif indéfini pour mabduu ${ }^{2} u n$ et en $-i$ du cas indirect défini pour suurati).

La question des terminaisons vocaliques est certainement la plus délicate. D'une part, c'est l'une des caractéristiques les plus marquantes de l'arabe littéraire et, d'autre part, c'est l'un des points sur lesquels l'AMS présente, à l'oral, le plus de variation $^{25}$. Ici nous ferons remarquer la cohérence de ce passage: les marques casuelles sont toujours réalisées à l'intérieur des syntagmes et jamais à la fin des syntagmes, on les trouve donc dans tous les noms N1 en fonction de déterminé (tartiibu, nuzuulu, tanazzulu et suurati) mais pour aucun des noms N2 en fonction de déterminant (-l-mushaf, -l-qur ${ }^{?} a a n$ et $\left.l-^{\varsigma} a l a q\right)$.

Il existe cependant des cas où les terminaisons sont réalisées avec un soin plus constant, ainsi dans ce passage, qui s'insère pourtant entre la particule yœgंsyr typiquement hassaaniyya et un membre de phrase resté interrompu auquel l'emprunt au "franglais" cow-boy donne une allure très dialectale:

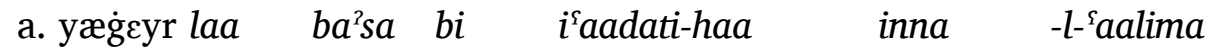 a. mais nég mal avec répétition-3Fs que le savant a. “[...] mais il n'y a pas de mal à le répéter, que le savant
b. laa yakuunu ${ }^{\text {} a a l i m a n ~ s a a l i h a n ~ i l l a a ~ i đ a a ~ k a a n a ~}$
b. nég est(3MS) savant véritable seulement si était(3MS)
b. "n'est un savant véritable que si son savoir
c. $m a^{\mathrm{S} a}$ 'ilmi-hi al-vițna ... i i uud kæḅḅıyyaat maa...
c. avec science-3MS la sagacité devient(3MS) cow-boys nég....
c. "est accompagné de sagacité,... de sorte que les bandits ne (peuvent)..."

Ici on notera que la prononciation très soignée (/a/ réalisé bien ouvert, durée plus nette des longues finales, ...) ne laisse subsister de la prononciation mauritanienne du classique que la sonorisation $\mathrm{du} / \mathrm{f} /$, cf. vițna pour fitna ${ }^{26}$. Ceci va de pair avec la présence de toutes les finales vocaliques, tant verbales que nominales - noter en particulier le -an de l'adjectif șaalihan, pourtant en fin de syntagme - à l'exception du terme final viṭna qui est à la forme pausale ${ }^{27}$.

${ }^{25}$ Ces variations dépendent bien sûr du degré de connaissance et de maîtrise de l'AMS, mais l'étude de D. B. Parkinson (1994) a montré aussi, de manière plus significative, qu'il y a des lois tendancielles dans l'apparition des finales vocaliques.

${ }^{26}$ La sonorisation de la labiale fricative est caractéristique du dialecte hassaaniyya (de Mauritanie du moins, car le trait est beaucoup moins net chez les hassanophones du Maroc), mais elle ne fait l'objet d'aucune stigmatisation, y compris semble-t-il pour la prononciation du classique où les prononciations correctes du žiim et du daad ont pourtant fait l'objet d'importants débats (voir par exemple L. Bouvat, 1913). Ceci est bien la preuve que l'AMS de chaque locuteur n'est pas indépendante du dialecte maternel (voir notamment à ce propos, A. Kaye, 1972: 44-5), même s'il ne s'agit ici que d'un problème de phonétique et non pas, comme ce peut être le cas ailleurs (en particulier avec les interdentales), d'un réel problème phonologique.

${ }^{27}$ Nous n'avons pas observé le - $h$ final qui devrait semble-t-il apparaître dans les terminaisons pausales du féminin, mais qui n'apparaît pourtant pas toujours (cf., pour la prononciation cairote, D. B. Parkinson, 1994). 


\section{L'alternance codique intra-propositionnelle}

Nous allons étudier maintenant des phénomènes d'alternance qui se produisent dans un cadre syntaxique plus limité et nous essayerons de voir si les contraintes observées généralement dans les cas de code-switching sont ou non respectées.

(3) a. đæ l-æwwæl faad və-l-axiir đæ lli mən aaxər

a. ce le premier est-devenu(3MS) dans-la fin ce qui à partir de fin

a. "Le premier est donné en dernier... Ce qui était à la fin

b. ši saad -ewwl-u mannaa-ha enn

b. chose est-devenu(3MS) dans début-3MS sens-3FS que

b. "a été placé au début... Cela signifie que

c. tartiib æl-mușhaf ruu siycet vii-h umuur

c. ordonnancement le Livre ont-été-observées(3FS) en-3MS choses

c. "dans l'ordonnancement du Livre on a pris en considération des éléments

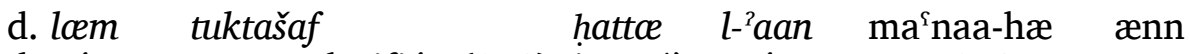

d. nég. se-sont-clarifiées(3FS) jusqu'à présent sens-3FS que

d. "qui sont restés non clarifiés jusqu'à présent. Cela signifie que

e. əl-qər̆aan mæ -zaal vaașəl əv mæddæ

e. le Coran nég. a-cessé(3MS) ayant-besoin(3MS) dans gens

e. "le Coran a encore besoin que des personnes

f. tșiib l-u tegraa-h u tædæbbr-u ilıyn

f. se-libèrent(3FS) pour-3MS lisent-3MS et méditent(3FS)-3MS jusqu'à

f. "prennent le temps de l'étudier et de le méditer pour

g. ta'ṛa əl-ḥikmæ vii 'uduuli s-sahạabati bi

g. savent(3FS) la sage-raison dans non-choix les Compagnons avec

g."connaître la raison qui explique le choix des Compagnons et

h. Piqraarin min rașuuli -llaahi șalla -llaahu

h. confirmation-une venant-de Envoyé Dieu a-prié(3MS) Dieu

h. "sa confirmation par l'Envoyé de Dieu, que Dieu prie

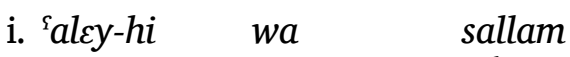

i. sur-3MS et a-donné-la-paix(3MS).

i. "pour lui et lui accorde la paix".

Cet extrait se termine par un passage relativement long en AMS, dont on remarquera surtout (en dehors du phénomène de pause qui écourte l'accompli de sallama) qu'il présente une alternance codique juste après le complément d'objet direct hikma - un emprunt à l'AMS qui apparaît sous une forme "médiane" -, au niveau du complément d'objet indirect vii ^uduuli ș-şahaabati dont la forme classicisée ne correspond à aucune nécessité syntaxique, mais ne viole pas non plus quelque règle que ce soit.

La même observation peut être faite pour les deux alternances codiques précédentes, sauf qu'il s'agit cette fois clairement d'insertions. Le fait que ces syntagmes appartenant à l'AMS soient insérés dans un contexte médian, et non pas purement dialectal, fait que les limites précises de l'alternance ne sont pas 
d'une grande netteté. Dans le cas de ruúriyat en c., on a affaire à un passif vocalique, donc à une pure forme d'AMS, mais son contexte (complément mis en relief tartiib al-mushaf ...vii-h et sujet postposé umuur) paraît plus médian que classique si on le compare avec le tartiibu -l-mushaf de l'exemple (1a). Quant au syntagme lam tuktašaf en d., si on peut hésiter à classer son complément hattce l'aan "jusqu'à maintenant" en AMS ou en arabe médian (en dialecte, et généralement en médian, on emploierait l'expression équivalente ilkyn đậ̣), il relève clairement de l'AMS, car le passif vocalique de VIIIè forme dérivée (sans équivalent en dialecte où les formes en $t$ - et -t- n'ont pas de passif) est à l'inaccompli apocopé, comme le veut l'emploi de la négation très classique lam.

Si ce passage, et surtout ce dernier exemple, illustre surtout les contraintes de compatibilité entre le verbe et ses morphèmes, celui qui suit, où les changements de code sont très nombreux, montre que l'unité d'alternance est tantôt le syntagme et tantôt la proposition:

(4) a. yuqaalu annæe ${ }^{\mathrm{s}}$ umar bin əl-xațaab radiyce

a. est-dit(3MS) que Umar fils al-Khattaab a-été-satisfait(3MS)

a. "On dit que [le calife] Umar bin al-Khattaab, que Dieu soit

b. allaahu ${ }^{\text {}} a n-h u$ ænn-u qara? $a$ u haađæ -1-kælaam

b. Dieu de-3MS que-3MS a-lu(3MS) et cette(MS) la parole

b."satisfait de lui, qu'il a lu ... Et ces propos

c. lli nguul əl-kum žaayb-u al-haafiđ̛̣ bni ${ }^{\text {sasaakir }}$

c. que dis(1S) à-2P apportant-3MS al-haafidh fils Asaakir

c. "que je vous cite, al-ḥaafidh bin Asaakir l'a rapporté [...]

d. šcy?un min haađaa u žaab-u -1-imaam al-qurțubi

d. une chose de cela et a-apporté(3MS)-3MS l'imam al-Qurtubi

d. "quelque chose [...], ainsi que l'imam al-Qurtubi,

e. muvassir əl-qวr? ${ }^{2}$ aan iđæn ællaa đækru-h 'ulamaa

e. exégète le-Coran ainsi seulement ont-cité(3P)-3MS savants

e. "l'exégète du Coran, qui a été cité lui-même par des savants auxquels on

f. $y u^{\varsigma}$ taddu bi-him naqlan wa ${ }^{\varsigma}$ aqlan wa

f. est-appuyé(3MS) avec-3MP transmission et raison et

f. "peut se fier pour la transmission et pour la spéculation, pour leur

g. fitnatan wa fahman ann xaalig šaabb

g. discernement et compréhension que existant(MS) jeune-homme

g. "finesse et leur intelligence... Il y avait donc un jeune homme qui,

h. sami ${ }^{\varsigma} a$ aayotan mən əl-qoriªan tutlaa

h. a-entendu(3MS) un-verset de le Coran est-récité(3FS)

h. "ayant entendu réciter à haute voix un verset du Coran,

i. va sa'iqa wa maatce wæ -'1-ænn-u

i. alors s'est-évanoui(3MS) et est-mort(3MS) et sur-que-3MS

i. "s'était évanoui puis était mort. Et c'est ainsi que, 


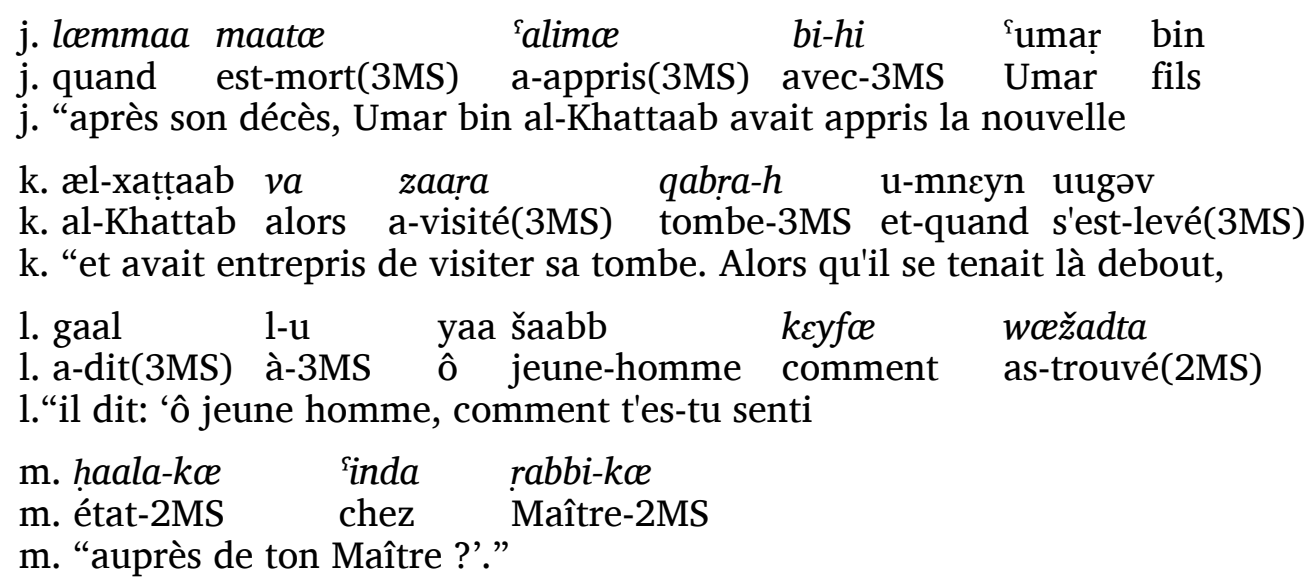

$\mathrm{Ne}$ pouvant commenter chaque forme, nous nous contenterons d'attirer l'attention sur quelques phénomènes parmi les plus saillants:

- la variation du pronom affixe de 3MS en fonction du contexte, à savoir des formes dialectales monophonématiques ( $h$ après voyelle, ex. đakru- $h$ en e.; $-u$ après consonne, ex. žaab-u en d., gaal $l-u$ en 1.), en alternance avec des formes classiques biphonématiques (-hu au cas sujet, ex. ${ }^{\S} a n-h u$ en b.; -hi au

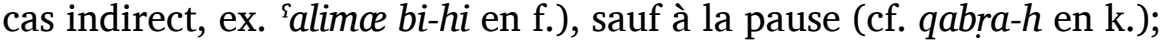

- une congruence entre le choix des conjonctions, des prépositions, etc..., et la présence ou non de terminaisons désinentielles (la particule $f a$ avec des accomplis en - $a$, cf. $v a$ ș $a^{\Upsilon} i q a$ en i. et $v a$ zaara en k.; les particules classiques lammoe et keyfae avec les accomplis classiques maatce et wažadta en j. et 1 ., mais la conjonction dialectale mncyn devant l'accompli dialectal uugəv en k.; la préposition classique 'inda au lieu de la préposition dialectale ${ }^{\text {s}}$ and devant le nom rabbi au cas indirect en $\mathrm{m}$.);

- des compléments d'objet au cas direct (ex. sæmi ${ }^{\uparrow} a$ aaycetan en h., zaara qabra$h$ en k., wažadta haala-kae en l.m.) et même des compléments de manière au

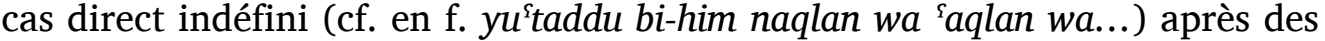
verbes conjugués comme en AMS, mais jamais après des verbes conjugués comme en dialecte.

Bien que l'alternance dialecte/variété standard ne pose pas les mêmes problèmes de compatibilité que l'alternance entre deux langues non apparentées, notamment parce que les problèmes d'ordre y sont beaucoup plus limités ${ }^{28}$ (cf. A. Giacalone Ramat, 1995: 56 et sq.), on peut donc conclure de ces exemples que l'alternance codique ne se fait pas pour autant en n'importe quel point de l'énoncé.

$28 \mathrm{Si}$ nous n'avons pas évoqué cet aspect jusqu'à présent, c'est parce que nous n'avons pas rencontré de différence sur ce point entre l'AMS et le ḥassaaniyya. 


\section{Les phénomènes de continuum}

Le seul exemple rencontré qui fasse plus ou moins exception à la règle et qui tende à présenter une alternance codique entre un morphème grammatical et le centre dont il dépend est le cas suivant.

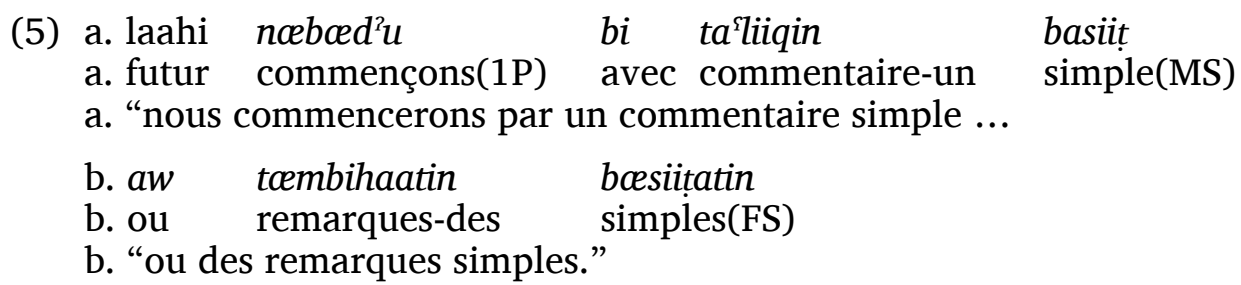

Avec le verbe bad $e^{2} \propto$ - synonyme du hassaaniyya sæentce "commencer" - on aurait peut-être pu trouver la particule de futur $s a$-, et non la particule dialectale laahi. Malgré la quasi identité des 1ères personnes de pluriel en dialecte et en AMS, il semble cependant que la forme ncebced ${ }^{2} u$ soit clairement conçue par le locuteur comme une forme "médiane" (compatible donc avec laahi), distincte de la forme classicisante nobd $e^{2} u$ (qui, elle, aurait pu nécessiter l'emploi de $s a$-).

Ceci nous donne l'occasion de revenir sur des phénomènes rencontrés dans les exemples précédents. Les syntagmes nominaux bi-l-faatiha en (1a.), $l$ - $^{\complement}$ alaq en (1d.), hasæb on-nuzuul en (1e.), al-vițna en (2c.) ou yaa šaabb en (41.), peuvent en effet être considérés (plus encore que nabœe ${ }^{2} u$ ), soit comme des formes classicisantes prononcées sans insistance sur la terminaison (tous les noms sont en fin de syntagme, sinon de proposition), soit comme des formes mixtes (du médian). Auparavant, faute de disposer de trois styles typographiques différenciés, nous avions choisi de classer ces formes en tenant compte du contexte immédiat, évitant de supposer une alternance là où nous n'avions pas de signe visible ${ }^{29}$. Mais il nous est peut-être arrivé de privilégier la proximité du dialecte avec l'arabe médian au détriment de celle qui existe entre le médian et l'AMS.

Si l'on considère le passage suivant, on se rend compte qu'il illustre bien le rôle de transition rempli par les formes hybrides de l'arabe médian. (Nous mettons ici entre parenthèses les groupes qui appartiennent plus spécifiquement à l'arabe médian et en italiques entre parenthèses toutes les formes mixtes susceptibles de se rencontrer à l'oral en AMS - du moins en prononciation courante.)

(6) a. mindṛa huwwæ (al-muṣhaf aš-šariif) budiªe bi mađaa

a. est-ce que lui le Livre le noble a-été-commencé avec-quoi

a. "je vous demande avec quoi le Livre noble a été commencé

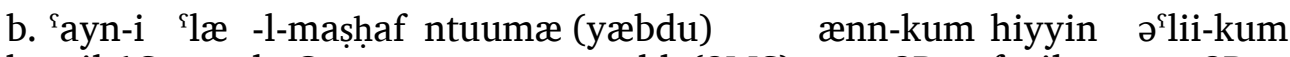

b. œil-1S sur le Coran vous semble(3MS) que-2P facile sur-2P

b. “je veux dire que le Coran, vous, il semble qu'il vous est facile

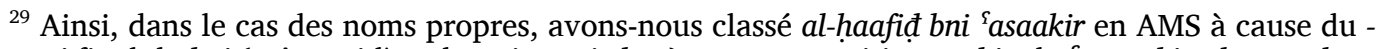

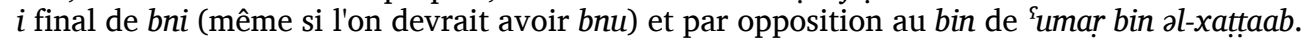



c. taxltu
wahdeyn gaalu
budi? $c$
(bə $\mathrm{qra}^{2}$ )
c. vous-trompez(2P) plusieurs ont-dit(3P) a-été-commencé avec lis(2MS)
c. "de vous tromper. Plusieurs ont dit: 'il commence avec le verbe "lis!"'.
d. aanæ maa gəlt (cewwalu maa) budi? $œ e$ bi-hi
d. moi nég. ai-dit(1S) premier-3MS rel. a-été-commencé avec-3MS
d. "Moi je n'ai pas dit: 'la première chose par laquelle a commencé
e. (cel-qur ${ }^{2} a a n$ fii-h varq beyna-maa) budi? $œ$ bi-hi
e. le Coran en-3MS différence entre-rel. a-été-commencé avec-3MS
e. "le Coran, car il y a une différence entre ce par quoi commence
f. (al-muṣhaf
wa maa) budi? ce
bi-hi
f. le Livre
et rel. a-été-commencé
avec-3MS le Coran
f. "le livre et ce par quoi a commencé le Coran."

Les deux formes pour lesquelles il y a quelques hésitations sont finalement deux formes verbales (ycbdu et qra $^{\text {}}$ ) qui, plus que les formes nominales, apparaissent comme des formes mixtes - elles seraient sans doute perçues comme fautives en $\mathrm{AMS}^{30}$.

D'un point de vue purement formel, on a donc là un usage assez spécifique de l'arabe médian et, par là même, des trois codes. On peut se demander si cela donne raison aux linguistes qui veulent voir dans la langue arabe non pas une diglossie, une triglossie, voire une multiglossie, mais un continuum. Si cette position semble difficile à soutenir sociolinguistiquement - notamment à cause des phénomènes de code-switching -, la variation constatée justifie l'exploration d'un nouveau point de vue, celui de la pragmatique et de la stylistique $^{31}$.

\section{LA VARIATION AU SERVICE D'UNE STRATEGIE DISCURSIVE}

Après avoir étudié en détail comment se fait la commutation, nous pouvons nous interroger sur le sens que prennent les changements de code chez O. S. Y.

\section{Le code de la complicité}

Le dialecte est le seul code que maîtrise effectivement l'auditoire attitré de O. S. Y. Certes, bon nombre d'expressions d'AMS, (notamment les adverbes comme tab ${ }^{\text {san }}$ "bien sûr" ou maetalan "par exemple", mais aussi une partie du vocabulaire politique comme ra iiis al-dowlee "le chef de l'Etat"), ont une fréquence telle dans les médias qu'elles sont susceptibles d'être utilisées par tous. Mais vis-à-vis de l'arabe médian dans son ensemble, le public habituel des prêches est dans une situation intermédiaire: s'il le maîtrise mal à la production,

30 On notera, à propos de muṣhaf, la variante typiquement dialectale maṣhaf qui apparaît justement dans un contexte très dialectal.

31 Si continuum il y a, c'est plutôt du côté de la stylistique qu'il faut le chercher, comme le suggère F. T. Mitchell qui considère que l'ESA, caractérisé par la non-stigmatisation, occupe un large spectre allant de l'informal casual au formal en passant par l'informal careful (cf. notamment son schéma, 1986: 17). Notre position, cependant, se rapprocherait plutôt de celle de G. Meiseles (1980) qui situe le "formel" (son "Sub-standard Arabic") du côté de l'AMS. 
il le maîtrise sans doute à peu près à la réception. Le hassaaniyya est donc, non seulement le code le plus familier à tous, mais aussi, pour O. S. Y. et son auditoire, la langue "de proximité", celle qui permet aux interlocuteurs de se sentir proches, en adéquation parfaite les uns avec les autres. Utilisé dans le contexte des prêches - où il ne correspond pas au code attendu -, ce code crée une complicité tout à fait particulière que O. S. Y. cherche consciemment à instaurer.

C'est ainsi notamment qu'on peut comprendre les différentes prises à partie directes que le prêcheur adresse à son public. Dans la cassette intitulée "Exégèse de la faatiha", par exemple, il précise que ses interventions ont lieu un lundi sur deux et il en profite pour parler du caractère béni de la nuit du lundi. Après avoir évoqué, en arabe classique, la vie du Prophète - en développant l'idée que les faits les plus marquants se ramènent à la nuit du lundi où il naquit - , il change à la fois de ton et de code pour demander en riant:

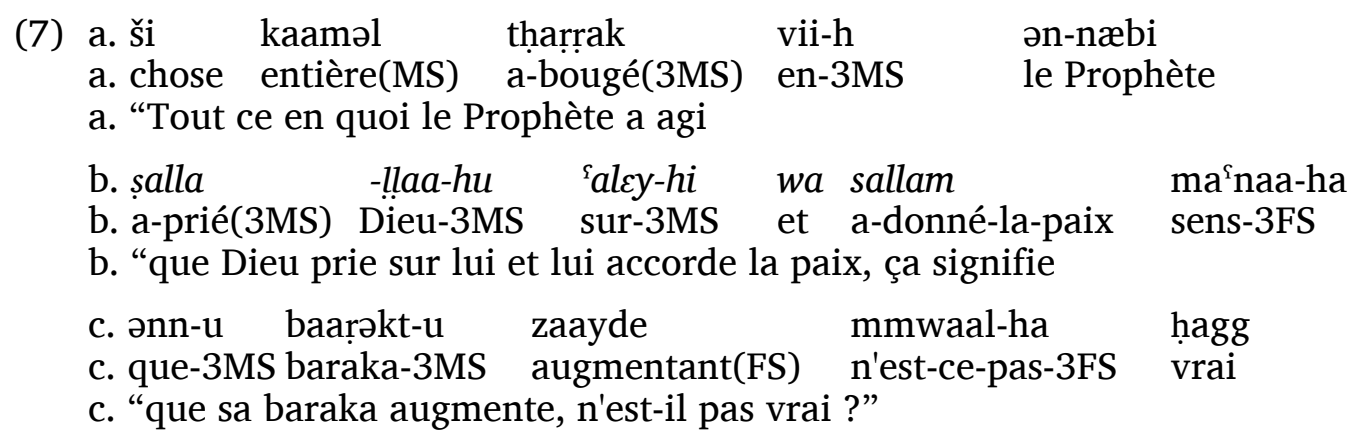

Après cette question, qui fournit l'occasion de résumer les phrases précédentes, le public est pris encore deux fois à partie. Il s'agit là plutôt de fausses interrogations, mais elles permettent à O. S. Y. d'interrompre son monologue, tout en montrant qu'il n'est pas seulement un livre qui parle, mais bien quelqu'un qui connaît ses interlocuteurs et leurs problèmes.

(8) a. walla maa-hi hagg walla vii-h qawleyn a. ou nég.-3FS vrai ou en-3MS opinion-duel

a. "Ou cela n'est-il pas vrai ? ou peut-on avoir là-dessus deux points de vue divergents ?"

On trouvera en (6) une autre interrogation rhétorique (6a.), suivie d'une fausse critique (6b.c.) - il feint de croire que son public pourrait ne pas savoir comment commence le Coran - qui fonctionne plutôt comme une taquinerie ou même un compliment indirect à son auditoire (peu suspect en fait d'ignorer une telle chose).

La complicité entre le prêcheur et son public se matérialise dans l'usage d'un hassaaniyya à l'accent et au registre nettement populaires. Au ton grave et solennel adopté en général pour l'AMS, succède une tonalité beaucoup moins sérieuse (du ton enjoué, voire rigolard, à un ton quasiment graveleux) qui met le public populaire à son aise. De ce point de vue, le style de O. S. Y. (qui appartient à une tribu de lettrés originaire de l'est du pays), relève d'un comportement beaucoup plus libre et décontracté que celui qu'on observe chez 
les lettrés du sud-ouest (celui-ci se caractérise par une extrême retenue dans la manière d'agir comme dans la manière de parler - lenteur du débit, ton monocorde, lexique choisi). S'adressant surtout à des hiraațiin du sud-ouest, $\mathrm{O}$. S. Y. parvient ainsi, par sa manière de parler le dialecte, à annuler la distance sociale qui le sépare de son public.

L'apparition, dans le hassaaniyya du prêcheur, d'expressions typiquement populaires, correspond elle aussi à un mouvement de l'orateur en direction de l'univers culturel de son auditoire. Ainsi, au début de cette même cassette consacrée à la faatiha, l'emploi surprenant du verbe gabb "s'abstenir de boire un jour sur deux (pour le bétail)", pour dire que, ne prêchant qu'un lundi sur deux, ils devront venir seulement une fois sur deux:
(9) a. ntæmmu
nžu
maṛa u ngubbu
sann-u marṛa
a. continuons(1P) venons(1P) fois et abstenons...(1P) de-3MS fois
a. "Nous venons une fois et nous nous abstenons de venir la fois suivante."

L'emploi, très inattendu, est encore souligné par O. S. Y. demandant à son public s'il sait ce que veut dire gabb (nouvel exemple de question on ne peut plus rhétorique !).

Dans une autre cassette, dont est extrait l'exemple (2), la rupture de ton après le passage en AMS s'opère avec kabbbcyyaat, qui appartient au hassaaniyya populaire des villes. Si cet emploi en lui-même ne fait qu'amorcer une proposition restée inachevée, le changement de code est souligné dans la phrase suivante par le recours à une expression dialectale qui permet à O. S. Y. de faire un jeu de mots sur azrag. Après avoir présenté un éminent savant, il dit de lui:

(10)a. tæmm

$$
\text { đ̣aann aẓrag w- aṣfar }
$$

a. a continué(3MS) pensant(MS) sur que-3MS moucheté et jaune

a. "On continue à croire qu'il est naïf et jaune

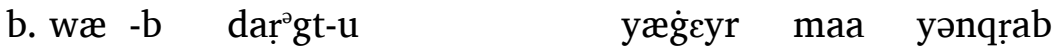

b. et avec tache-sur-front-3MS mais nég. est-approché

b. "avec sa tache sur le front, alors que sa brillante

c. $m a^{\text {}} a$ maa rivati al-ahkaami al-vațin

c. avec connaissance les règles-de-droit la-sagace(MS)

c. "connaissance des règles du droit le rend redoutable."

Ces exemples sont caractéristiques de la fonction spécifique dévolue au hassaaniyya dans les prêches de O. S. Y. Au-delà d'une parfaite compréhension, il s'agit d'établir une complicité sociale, de faire rire et d'en profiter pour se moquer des ennemis politiques qui, bien entendu, sont désignés régulièrement par des métaphores ridiculisantes telles que 'ulcemaa banaayce "les théologiens du casse-croûte ${ }^{1132}$ - ainsi nommés pour stigmatiser la corruption des oulémas (cf. Z. O. Ahmed Salem, 1996: 250).

\footnotetext{
${ }^{32}$ Le registre de banaayæe, terme dialectal d'origine mystérieuse, contraste avec celui de ${ }^{\S} u l œ m a a$.
} 


\section{Le code de la distance}

Bien que l'AMS soit d'autant plus éloigné du dialecte que les finales désinentielles sont prononcées, certains passages en pur arabe classique ne posent pas vraiment de problème de compréhension. Les formules telles que salla -llaa-hu 'alcy-hi wa sallam sont en effet connues de tous. Pour le reste, l'auditoire de O. S. Y. est incapable de suivre un discours (religieux ou non) qui se ferait entièrement en AMS. L'une des premières raisons du succès de notre prêcheur tient donc au fait qu'il est l'un des seuls à parler autant en dialecte durant ses prônes. Cette restriction n'est pas due chez O. S. Y. à une quelconque difficulté à s'exprimer en AMS. Bien au contraire, il apparaît à tout un chacun et plus encore aux personnes instruites - que O. S. Y. s'exprime avec beaucoup d'aisance en arabe classique. Puisque le public populaire ne peut pas goûter un discours réalisé uniquement en AMS, il va s'agir, pour O. S. Y., de se limiter au minimum et d'en tirer le meilleur parti possible.

La stratégie adoptée consiste en général à commencer le prône en AMS, avec des références au Coran et à la vie du Prophète - ce qui permet d'instaurer une ambiance sérieuse teintée de religiosité. Dans le cas des cassettes, on notera que cette connotation est donnée dès le titre (tafsiir cel-fatiiha par exemple), mais que la référence fréquente à une localité (asỉilaat dyigæenni "les questions de Djigenni") est une manière de particulariser les discours en les ancrant dans une réalité moins abstraite. Cette manière très habile de poser la distance, pour ensuite la réduire partiellement, est assurée dans le prêche par l'abandon de l'AMS au profit d'un arabe plus dialectal. L'alternance est d'autant moins problématique que la plus grande partie des prêches est constituée d'anecdotes. Celles-ci font intervenir des personnages célèbres de l'historiographie islamique, puis s'écartent petit à petit du point de départ pour se perdre dans d'innombrables digressions.

Ce qui est constant, en tout cas, ce sont les mouvements réguliers, quasi pendulaires, qui déclenchent des "remontées" de classicisation, tout particulièrement lorsque $\mathrm{O}$. S. Y. évoque des figures célèbres de l'Islam. En parlant d'eux en AMS, O. S. Y. se met de leur côté et profite de leur gloire pour asseoir sa propre position.

Ce constant équilibre à maintenir entre l'AMS, dont la maîtrise est signe de connaissance et d'esprit religieux, et le dialecte, qui permet le contact avec le public, fait que O. S. Y. est obligé de passer constamment de l'un à l'autre. Le faisant, il est obligé de limiter les alternances trop brutales qui casseraient le mouvement général du discours, si elles n'étaient réservées, outre les cas de citations, à des mises en valeur vraiment signifiantes. Ainsi, dans l'exemple suivant, il s'agit manifestement pour O. S. Y. de se poser lui-même en savant, en évoquant ceux qui maîtrisent réellement le Coran:

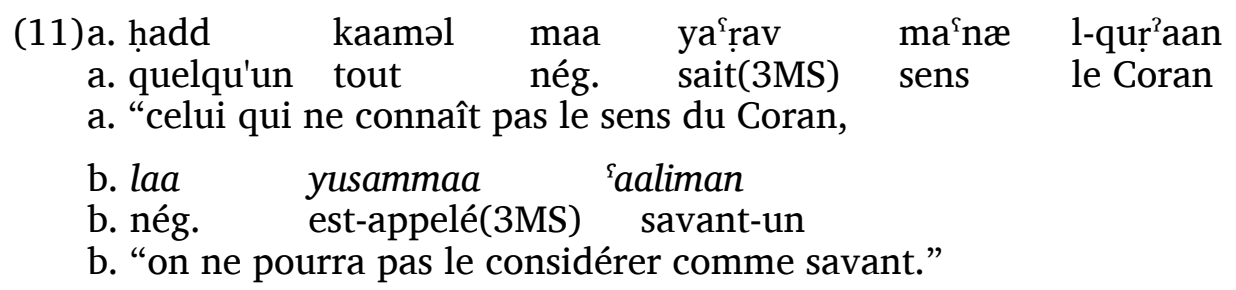


Quand les allers-retours entre le pôle AMS et le pôle dialectal sont progressifs, ils se réalisent à travers l'emploi de l'arabe médian. On peut donc dire que les formes mixtes ont essentiellement une fonction de transition $^{33}$ et qu'elles sont stylistiquement neutres.

\section{Deux codes plutôt qu'un}

Les changements codiques que nous avons étudiés jusqu'à présent sont socialement significatifs. Ils prennent tout leur sens du fait de l'association régulière qui existe dans la société entre les deux codes et des domaines d'activité plus ou moins spécifiques. Il y a cependant dans les prêches d'autres commutations dont la fonction est liée plus directement à l'organisation discursive.

Les études sur le code-switching ont montré que l'alternance codique avait tendance à devenir plus fréquente dans certains "lieux" conversationnels: en discours rapporté, en cas de changement de récepteur, dans les parenthèses explicatives, dans les réitérations, lors des changements d'activité, de sujet, de locuteur, etc. (cf. P. Auer, 1995: 120).

Dans les prêches de O. S. Y., nous avons trouvé quelques exemples de réitération. Ce peut être une simple reprise-traduction comme dans l'exemple (3) où la reformulation en dialecte (đce lli man aaxar ši ${ }^{\wedge} a a d v-e w^{w} l-u$ ) est équivalente à la formulation précédente en arabe médian (đœ l-œwwœl ईaad va-laxiir). Mais il s'agit le plus souvent d'une paraphrase plus libre, comme en (7), et on ne peut donc pas dire que les réitérations avec alternance de code soient caractéristiques des prêches étudiés.

Par contre, d'autres commutations sont frappantes, ainsi dans le passage donné en (4), où on a l'impression que les changements de code sont utilisés pour distinguer les plans du récit, annoncer les événements importants ou marquer le début du discours rapporté.

L'adoption du dialecte en b. (u haađa-l-kcelaam lli nguul al-kum) et en d. (u $\check{z} a a b-u$ ) fait une rupture - moins nette en b. à cause de la forme médiane kalaam - avec ce qui précède ( qra $^{2} a$ et $\check{s ̌ c y}^{2} u n$ min haađaa) et introduit la chaîne de transmission des auteurs appelés comme garants. Le changement de code, qui marque un commentaire, est l'équivalent à l'oral de l'ouverture d'une parenthèse.

Dans la suite du même extrait, le choix du dialecte annonce à chaque fois une nouvelle étape dans la narration: l'introduction d'un second personnage en g. (œenn xaalig šaabb), le retour au premier en i. (wœe-'l-œenn-u), la rencontre entre les deux et le début du dialogue en k.l. (u mncyn uugəv gaal l-u). L'alternance, généralement très rapide, fait que les éléments importants du récit et les paroles rapportées sont toutes en AMS (sæmi ${ }^{\Upsilon} a$ aayœtan en h.; va șa iqa wa maatce en i.; loemmaa maatce ${ }^{\complement} a l i m c e$ bi-hi en j.; va zaara qabra-h en k.; keyfoe wcežadta ... en 1.).

Cette organisation structurelle du discours, qui joue sur l'opposition entre les codes, est une caractéristique assez nette des prêches de O. S. Y. Dans cette même cassette (intitulée as illatu chli ațaar "les questions des gens d'Atar"), qui

33 A. Giacalone Ramat aborde dans son article (1995: 59) la notion de "neutral sites" qui favorisent l'alternance codique. Mais peut-être s'agit-il moins ici, avec les termes hybrides de médian, de favoriser le code-switching que de le rendre possible. 
est censée traiter de magie noire et de l'impossible résurrection des morts, on trouve la description d'une autre rencontre entre un vivant et un mort. Il s'agit cette fois d'un certain 'abdallah ibn 'umar qui est appelé par son nom, alors qu'il traverse le cimetière des non-musulmans morts à la bataille de Badr. Pour accentuer l'aspect solennel et tragique de la scène - un mort dont seul le haut du corps dépasse de la tombe - les éléments les plus forts du récit sont mis en relief par la commutation vers l'AMS. Ce sont aussi bien les paroles du mort $\mathrm{cf}$. (12), que la description de son corps cf. (13), le récit de la rencontre cf. (14) ou les commentaires sur cette rencontre cf. $(15)^{34}$.

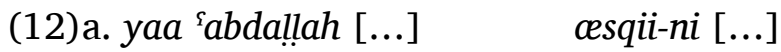

a. "ô Abdallah [3 fois], donne-moi à boire [3 fois]"

(13)a. iđaa bi silsilatin vi 'unuqi-hi

a. ainsi avec chaîne-une dans cou-3MS

a. "le voici avec une chaîne au cou"

(14)a. beyncemaa gaal l-u anaa amurru bi žaanib

a. tandis que a-dit(3MS) à-3MS moi suis-passé(1S) à côté

a. "il dit: 'tandis que je passais à côté..."”

(15)a. wæ huwwæ yunaadi wæ aanæ laa ${ }^{\text {}}$ aarifu-h

a. et lui a-appelé(3MS) et moi nég. connaissant(MS)-3MS

a. "alors il a appelé, mais moi je ne le connaissais pas".

O. S. Y. racontant les anecdotes avec force retours en arrière et répétitions, on se rend compte qu'il revient plusieurs fois sur les mêmes points forts du récit, mais qu'il maintient l'attention de l'auditoire grâce à de légères variations: changement du verbe exprimé en AMS, l'ancien prenant une forme dialectale cf. (16) ou ajout d'un nouvel élément, cf. "feu" en (17).

(16) a. agit-ni ag̈it-ni sgii-ni sgii-ni

a. secours(2MS)-1S [2 fois] abreuve(2MS)-1S [2 fois]

a. "au secours! au secours! donne-moi à boire ! donne-moi à boire !"

(17) a. đaak lli traa ${ }^{\text {i }}$ silsilatun mince -l-naari

a. cela rel. vois(2MS) chaîne-une en le feu

a. "ce que tu vois, c'est une chaîne de feu"

Il sait aussi bien être convaincant que passionnant et cela explique son succès, non seulement devant son public attitré, intéressé par ses idées, mais aussi auprès de l'auditoire plus large de ses cassettes, sensible à son talent oratoire.

On notera en (15) une montée graduelle du médian (yunaadi) au classique (laa ${ }^{\complement}$ aarifu-h) avec un pronom dialectal aanæ, qui contraste avec la forme classique anaa employée juste devant la forme verbale d'AMS amuṛ̣u en (14). 


\section{CONCLUSION}

L'étude proposée ici lève un voile sur la technique conversationnelle utilisée par O. S. Y. Il apparaît que la variation linguistique est mise au service d'une véritable stratégie discursive et que les alternances codiques correspondent à des choix motivés.

D'un certain point de vue on pourrait penser que les variations observées dans les prêches sont inséparables de la visée communicationnelle personnelle de O. S. Y. et qu'elle ne présente d'intérêt que pour l'étude de son style. Mais on peut voir que ce style est intimement lié aux potentialités et aux valeurs particulières que les variétés de l'arabe offrent aux locuteurs mauritaniens, selon leur appartenance sociale et leur degré d'instruction.

Cette étude d'un corpus original, relativement spécifique, permet d'approfondir notre connaissance des usages actuels de l'arabe, dans une perspective qui a déjà fait ses preuves pour l'analyse de discours politiques (cf. D. Holes, 1993, et N. Mazraani, 1995), mais dont on sait depuis longtemps (cf. El-Hassan, 1978) qu'elle est susceptible de s'appliquer aux divers types de discours réputés "formels" - sermons compris —. Notre approche permet notamment de montrer que les phénomènes stylistiques s'appuient à la fois sur l'existence d'une variété intermédiaire, aux formes hybrides, qui sert de code neutre, et sur l'existence de deux autres variétés, sociolinguistiquement bien différenciées, avec lesquelles l'alternance codique joue de manière signifiante.

Enfin, d'un point de vue plus général, on constatera que, si les phénomènes de code-switching entre dialecte et langue standard présentent de grands points communs avec ceux qu'on observe entre deux langues non apparentées, ils ont quand même la particularité de se combiner facilement avec des phénomènes de code-mixing. On peut donc avoir une image de continuum qui ne rend qu'imparfaitement compte du tableau des variétés linguistiques et des choix stratégiques que leur diversité permet. 


\section{REFERENCES}

Abd-el-Jawad, Hassan R. 1986. "The emergence of an urban dialect in the Jordanian urban centers". International Journal of the Sociology of Langage 61. 53-63.

Aguadé Jordi, Patrice Cressier \& Angeles Vicente éds. 1998. Peuplement et arabisation au Maghreb occidental. Madrid-Zaragoza: Casa de Velazquez \& Université de Zaragoza.

Auer, Peter. 1995. "The pragmatics of code-switching: a sequential approach". One speaker, two languages éd. par Lesley Milroy \& Pieter Muysken. Cambridge: Cambridge University Press. 115-135.

Blanc, Haïm. 1960. "Style variations in spoken Arabic: a sample of interdialectal educated conversation". Contributions to Arabic Linguistics éd. par Charles A. Ferguson. Cambridge, Mass.: Harvard University Press. 80-156.

Bouvat, L. 1913. "Un essai de Cheikh Sidia, sur la prononciation rituelle du "Dâd" et du "Dhâ". Revue du Monde Musulman 23. 305-314.

Cohen, David. 1963. Le dialecte arabe hassaaniiya de Mauritanie. Paris: Klincksieck.

Colin, Georges S. 1986. "Al-Maghrib. Aperçu linguistique". Encyclopédie de l'Islam vol. V. Nouvelle éd. Paris: Maisonneuve \& Larose. 1193-8.

El-Hassan, S. A. 1978. "Educated Spoken Arabic in Egypt and the Levant: A Critical Review of Diglossia and Related Concepts". Archivum Linguisticum s.d. 8:2. 112-132.

Ferguson, Charles. A. 1959. "Diglossia". Word 15. 325-340.

Giacalone Ramat, Anna. 1995. "Code-switching in the context of dialect/standard language relations". One speaker, two languages éd. Lesley Milroy \& Pieter Muysken. Cambridge: Cambridge University Press. 45-67.

Hawkins, Peter. 1983. "Diglossia Revisited". Language Sciences 5:1. 1-20.

Heath, Jeffrey. 1989. From code-switching to borrowing: foreign and diglossic mixing in Moroccan Arabic. London \& New York: Kegan Paul international.

Holes, Clive. 1993. "The Uses of Variation: A Study of the Political Speeches of Gamal Abd al-Nasir". Perspectives on Arabic Linguistics V éd. par Mushira Eid \& Clive Holes. Amsterdam \& Philadelphia: John Benjamins. 13-46.

Ibrahim, Muhammad H. 1986. "Standard and prestige language: a problem in Arabic sociolinguistics". Anthropological Linguistics 28. 115-126

Kaye, Alan S. 1972. "Remarks on Diglossia in Arabic: Well-defined vs. Illdefined". Linguistics 81. 32-48.

1994. "Formal vs Informal in Arabic: Diglossia, Triglossia, Tetraglossia, etc., Polyglossia - Multiglossia Viewed as a Continuum". Zeitschrift für Arabische Linguistik 27. 47-66.

Marçais, William. [1930. "La diglossie arabe", L'Enseignement public 12. 401-409. Repris dans] 1961. Articles et conférences. Paris: Adrien-Maisonneuve. 83-88.

Mazraani, Nathalie. 1995 "Functions of Arabic Political Discourse: The Case of Saddam Hussein's Speeches". Zeitschrift für Arabische Linguistik 30. 22-36.

Mitchell, T. F. 1980. "Dimensions of style in a grammar of educated spoken Arabic". Archivum Linguisticum s.d. 11: 2. 89-106. 
1986. "What is educated spoken Arabic?". International Journal of the Sociology of Language 61. 5-32.

Meiseles, Gustav. 1980. "Educated spoken Arabic and the Arabic language continuum". Archivum Linguisticum s. d. 11:2. 118-143.

Monteil, Vincent, 1948. Notes sur les Tekna. Paris: Larose.

Norris, H. T., 1986. The Arab Conquest of the Western Sahara, Harlow \& Beirut: Longman \& Librairie du Liban.

O. Ahmed Salem, Zakariya. 1996. Retour sur le politique par le bas. De quelques modes populaires d'énonciation du politique en Mauritanie. Thèse de sciences politiques, Université Lumière-Lyon II, Lyon.

O. Cheikh, Abdel Wedoud. 1985. Nomadisme, Islam et Pouvoir politique dans la société maure précoloniale (XIè siècle-XIXè siècle). Essai sur quelques aspects du tribalisme. Doctorat en sociologie, Université Paris V-R. Descartes, Paris.

1991. "La tribu comme volonté et comme représentation. Le facteur religieux dans l'organisation d'une tribu maure les awlâd Abyayri". Al-Ansâb la quête des origines éd. par P. Bonte, E. Conte, C. Hamès \& A.W. O. Cheikh. Paris: éd. de la MSH. 201-238.

Palva, Heikki. 1969. "Notes on classicization in Modern Colloquial Arabic", Studia Orientalia XL: 3. 3-41.

1982. "Patterns of Koineization in Modern Colloquial Arabic", Acta Orientalia 43. 13-32.

Parkinson, Dilworth B. 1994. "Speaking fuṣhaa in Cairo: the role of the ending vowels", Arabics Sociolinguitics: Issues and Perspectives éd. par Yasir Suleiman. Surrey: Curzon. 179-211.

Taine-Cheikh, Catherine (C. T.-C). 1978. L'arabe médian parlé par les arabophones de Mauritanie - Etude morphosyntaxique. Thèse de 3è cycle, Université Paris V-R. Descartes, Paris.

— 1983. "Le passif en hassaaniyya", Matériaux arabes et sudarabiques 1. 61-104.

-1988. "Métathèse, syncope, épenthèse: à propos de la structure prosodique du hassaniyya, Bull. de la Soc. de Ling. de Paris 83: 1. 213-252.

- 1988-98. Dictionnaire Hassaniyya-Français. 8 vol. Paris: Lib. Orientaliste Paul Geuthner.

- 1989. "La Mauritanie en noir et blanc. Petite promenade linguistique en hassaaniyya". Revue du Monde Musulman et de la Méditerranée 54: 4. 90-105.

- 1991a. "Le vent et le devant. De l'orientation chez les Maures". Journal asiatique 279: 1-2. 93-126.

- 1991b. "L'arabe des Bidân, un dialecte bédouin de Maghreb occidental". Semitics Studies (In honor of Wolf Leslau) éd. par Alan S. Kaye. 1991: 2. Wiesbaden: Otto Harrassowitz.1528-48.

- 1995-96. "Trois points de vue sur la négation maa dans le dialecte arabe de Mauritanie", Matériaux arabes et sudarabiques 7. Nouvelle série. 11-61.

- 1997. "Les hassanophones du Maroc. Entre affirmation de soi et autoreniement". Peuples Méditerranéens ("Langues et stigmatisation sociale au Maghreb") 79. 85-102.

- 1998. "Langues, savoirs et pouvoirs en milieu maure". Nomadic Peoples (Savoirs et pouvoirs. Formation et transformation des élites du monde nomade saharo-sahélien), éd. par Pierre Bonte \& Hélène Claudot-Hawad. 2: 1. Nouvelle série. 215-234. 
- 1998-1999. "Classement des dialectes arabes par rapport à deux macrodiscriminants". Matériaux arabes et sudarabiques 9. Nouvelle série. 11-50.

- 1999. "Le zénaga de Mauritanie à la lumière du berbère commun". Papers from the 9th Italian Meeting of Afroasiatic (Hamito-Semitic) Linguistics. Trieste 23 - 24 Aprile 1998, éd. par Marcello Lamberti \& Livia Tonelli. Padova: Unipress. 299-324.

Trudgill, Peter. 1986. Dialects in Contact. London: Basil Blackwell.

Wensinck, A J. 1986. "Khuṭba". Encyclopédie de l'Islam vol. V. Nouvelle éd. Paris: Maisonneuve \& Larose. 76-7.

Youssi, Abderrahim. 1983. "La triglossie dans la typologie linguistique". La Linguistique 19: 2. 71-83. 